SUBJECT AREAS:

ELECTRONIC PROPERTIES

AND DEVICES

OPTICAL PROPERTIES AND

DEVICES

Received

10 September 2014

Accepted

12 December 2014

Published

14 January 2015

Correspondence and requests for materials should be addressed to M.G. (martin.geller@ uni-due.de)

* These authors contributed equally to this work.

\section{Electron-beam induced nano-etching of suspended graphene}

\author{
Benedikt Sommer*, Jens Sonntag*, Arkadius Ganczarczyk, Daniel Braam, Günther Prinz, Axel Lorke \\ \& Martin Geller
}

Fakultät für Physik and CENIDE, Universität Duisburg-Essen, Duisburg 47048, Germany.

Besides its interesting physical properties, graphene as a two-dimensional lattice of carbon atoms promises to realize devices with exceptional electronic properties, where freely suspended graphene without contact to any substrate is the ultimate, truly two-dimensional system. The practical realization of nano-devices from suspended graphene, however, relies heavily on finding a structuring method which is minimally invasive. Here, we report on the first electron beam-induced nano-etching of suspended graphene and demonstrate high-resolution etching down to $\sim 7 \mathrm{~nm}$ for line-cuts into the monolayer graphene. We investigate the structural quality of the etched graphene layer using two-dimensional (2D) Raman maps and demonstrate its high electronic quality in a nano-device: A $25 \mathrm{~nm}$-wide suspended graphene nanoribbon (GNR) that shows a transport gap with a corresponding energy of $\sim 60 \mathrm{meV}$. This is an important step towards fast and reliable patterning of suspended graphene for future ballistic transport, nano-electronic and nano-mechanical devices.

G raphene as a two-dimensional lattice of carbon atoms $\mathrm{s}^{1,2}$ with its linear dispersion relation promises rich and interesting physical characteristics ${ }^{3,4}$ as well as devices with exceptional electronic properties, such as graphene-based transistors ${ }^{5,6}$ or electronic components ${ }^{7}$ which could be also based on ballistic transport ${ }^{8}$. However, the presence of an underlying supporting substrate for the delicate single atomic layer is a major drawback as it decreases the envisioned high mobility by scattering, induced by potential fluctuations ${ }^{9}$. This limiting factor is particularly relevant for $\mathrm{SiO}_{2}$ as the commonly used supporting material and drives the search for different materials, with hexagonal boron nitride (h-BN) presently being the most promising (but technically challenging) alternative ${ }^{10}$. However, the ultimate, truly two-dimensional system is still a freely suspended graphene layer without contact to any substrate ${ }^{11}$. The practical realization of nano-devices from suspended graphene, however, relies heavily on finding a structuring method which is minimally invasive and ensures an intact lattice structure.

Electron-beam lithography (EBL) followed by reactive ion etching is the most common technique for nanopatterning of supported graphene on insulating substrates into different structures like Hall-bars ${ }^{1,12}$, nanoribbons $(\mathrm{GNR})^{13,14}$, or quantum dots ${ }^{15,16}$. Resistless patterning techniques are an interesting alternative as they may reach higher resolution and are the only possible method for patterning suspended graphene, where the resist would otherwise damage the suspended structure. A focused-ion-beam (FIB) provides an easy and fast method for graphene processing on the nanoscale using Ga-Ions ${ }^{17}$ or He-Ions ${ }^{18,19}$. Although a resolution comparable to that of EBL can be achieved and it can be used for suspended graphene, the structural damage by energetic ion bombardment is a main drawback of FIB patterning ${ }^{20}$. The only second method for direct patterning of suspended graphene has been developed in a transmission electron microscope (TEM) ${ }^{21,22}$, however, no device fabrication has been demonstrated yet. Other techniques, which were developed for direct patterning of supported graphene only, are scanning tunneling microscope (STM) lithography ${ }^{23}$, atomic force lithography using anodic oxidation lithography ${ }^{24,25}$. Gas-assisted electron beam (FEB) etching is an alternative technique for direct patterning and has been used before with various precursor gases on different materials ${ }^{26,27}$, including $\mathrm{Si}^{28}, \mathrm{GaAs}^{29}$ and carbon ${ }^{30}$. It has also recently been used for direct etching of graphene layers that were supported on $\mathrm{SiO}_{2}$ with a resolution of better than $20 \mathrm{~nm}^{31}$. However, no direct FEB etching of suspended graphene into an electrical device has been demonstrated yet.

Here, we demonstrate high resolution, room temperature, nano-etching of suspended graphene layers into a graphene device. We use a scanning electron microscope (SEM) together with a selective carbon etch gas injection system, which uses water vapour as precursor gas (see methods). We demonstrate a patterning resolution down to $\sim 7 \mathrm{~nm}$ for line-cuts into the monolayer graphene and confirm the high quality of patterned graphene in two- 
dimensional (2D) Raman maps and transport measurements in a patterned nano-device. We also performed nano-etching on graphene layers, supported on $\mathrm{SiO}_{2}$, and show that this method works on single layer or bilayer graphene with a resolution down to $15 \mathrm{~nm}$ (see supplementary information).

\section{Results and Discussion}

Figure 1 shows nano-etching of a suspended graphene layer. Figure 1(d) depicts schematically the sample design, where the suspended graphene layer was contacted by two Au contacts that are separated by $500 \mathrm{~nm}$. The Au contacts were defined by standard electron-beam lithography (EBL). Afterwards, the $\mathrm{SiO}_{2}$ was selectively etched by hydrofluoric acid (see methods for more details). In Fig. 1(a) an SEM image of such a suspended graphene layer with a single line-cut is shown, while Fig. 1(b) displays a zoom-in of the area in 1 (a) marked by a white box. For these samples, the acceleration voltage was set to $\mathrm{V}=30 \mathrm{kV}$ and a minimal dose of $4 \mu \mathrm{C} / \mu \mathrm{m}^{2}$ was chosen to obtain a high-resolution incision with an average width of only $\sim 7 \mathrm{~nm}$ across the entire graphene sheet. This is the maximum resolution achieved so far using this gas-assisted electron beam etching technique on graphene. The patterning resolution for suspended graphene is a factor of two better than for graphene deposited on $\mathrm{SiO}_{2}$ (see supplementary information), as there are less secondary and backscattered electrons from the substrate that decrease the nominal resolution of the primary electron beam (proximity effect). Finally, Fig. 1(c) demonstrates an example of how this high-resolution patterning can be used for the fabrication of nanostructured graphene devices. We patterned a suspended graphene nanoribbon having a width of $20 \mathrm{~nm}$ and a length of $200 \mathrm{~nm}$. Note here, that the areas that were cut-off to from the graphene flakes roll-up to small graphene tubes with a diameter of approximately $30 \mathrm{~nm}$ on top of the graphene nano-ribbon, demonstrating another possibility of using this technique for advanced high-resolution patterning of various graphene nanostructures.

In the following, we concentrate (i) on Raman spectroscopy to demonstrate the high material quality of the patterned graphene layers and (ii) transport measurements on a first graphene device: a $25 \mathrm{~nm}$-wide suspended GNR. Figure 2 presents the quantitative analysis of the defect concentration in a suspended graphene layer after etching a $\sim 50 \mathrm{~nm}$-wide line-cut. The line-cut is visible in an SEM image shown in Fig. 2(a). We used room temperature Raman spectroscopy in a micro-photoluminescence ( $\mu$-PL) set-up with a scanning unit (see methods for more details) to record Raman spectra every $100 \mathrm{~nm}$ on the suspended layer. Figure 2(b) compares a Raman spectrum before and after etching. Before the etching step, a distinct fingerprint of a single layer of graphene is visible with a symmetric $2 \mathrm{D}$ peak higher in intensity than the $\mathrm{G}$ peak and no presence of a D-type peak, which is associated with defects in single layer graphene ${ }^{33}$. After etching, the $2 \mathrm{D}$ peak is still visible, however, reduced in intensity but still symmetric. Also, the defect peak D appears at a Raman shift of $\sim 1335 \mathrm{~cm}^{-1}$. We observe a higher intensity of the $2 \mathrm{D}$ peak for suspended graphene than for the supported graphene on $\mathrm{SiO}_{2}$, see Fig. 2(b). This lower 2D intensity of supported graphene is due to charged impurity scattering, a process that has been studied before by using Raman spectroscopy ${ }^{32}$.

To obtain a quantitative number for the defect density originated from the patterning, we use the model proposed by Cancado and coworkers $^{34}$. In this model the authors calculate the average distance between two point defects from the ratio of the $D$ and $G$ peak intensity, $I_{D} / I_{G}$, and the laser energy used for the measurement for a low defect density. Figures 2 (c) and (d) show the Raman maps for the 2D peak and the $\mathrm{I}_{\mathrm{D}} / \mathrm{I}_{\mathrm{G}}$ ratio, respectively. One of the two line-cuts is even visible in the intensity map of the $2 \mathrm{D}$ peak of the left suspended graphene layer between two areas of higher 2D intensity. Still, after the etching process, the $2 \mathrm{D}$ peak intensity is higher than the corresponding intensity of the supported (but unetched) graphene, visible at the left and right edge of this Raman map. This supports our assessment that the quality of the suspended graphene stays very high using the electron-induced etching method.

The Raman intensity ratio $\mathrm{I}_{\mathrm{D}} / \mathrm{I}_{\mathrm{G}}$ obtained from the $2 \mathrm{D}$ Raman map in Fig. 2(d) shows, however, that the etched graphene flake has an increased number of defects. We take now the average peak-height ratio $\mathrm{I}_{\mathrm{D}} / \mathrm{I}_{\mathrm{G}}$ for seven neighbouring scans, determined by single Lorentzian line fits to the D peak and the combined $G$ and $\mathrm{D}^{\prime}$ peak (which we cannot separate and fit using a single Lorentzian line) in the marked area in Fig. 2(c), the suspended graphene layer on the right-hand side. Plotting this ratio versus the position perpendicular to the etched cut, shows an increase of $\mathrm{I}_{\mathrm{D}} /$ $\mathrm{I}_{\mathrm{G}}=1.2$ at the bottom edge of the graphene layer up to $\mathrm{I}_{\mathrm{D}} / \mathrm{I}_{\mathrm{G}}=1.8 \mathrm{in}$ the middle, where the graphene has been nano-etched (black line in Fig. 2(e)). On the other side of the cut, the intensity ratio drops again down to $\mathrm{I}_{\mathrm{D}} / \mathrm{I}_{\mathrm{G}}=1.1$ near the upper edge of the graphene sheet. From this intensity ratio profile, we are able to allocate a point defect density to the so-called low defect density area (stage 1, see Ref. 34 for more details), where the ratio of $\mathrm{I}_{\mathrm{D}} / \mathrm{I}_{\mathrm{G}}$ increases with increasing number of defects. If the patterning caused higher defect densities (stage 2), we would expect from Ref. 34 that the $\mathrm{I}_{\mathrm{D}} / \mathrm{I}_{\mathrm{G}}$ ratio decreases from the graphene edges to the middle of the flake, where the etched line-cut is located (for a displacement of $\sim 1.3 \mu \mathrm{m}$ in Fig. 2(e)). If we passed from stage 1 to stage 2 while scanning over the graphene flake, the intensity ratio would have increased and dropped again, i.e. we would have seen a minimum in the $\mathrm{I}_{\mathrm{D}} / \mathrm{I}_{\mathrm{G}}$ profile near the etched line-
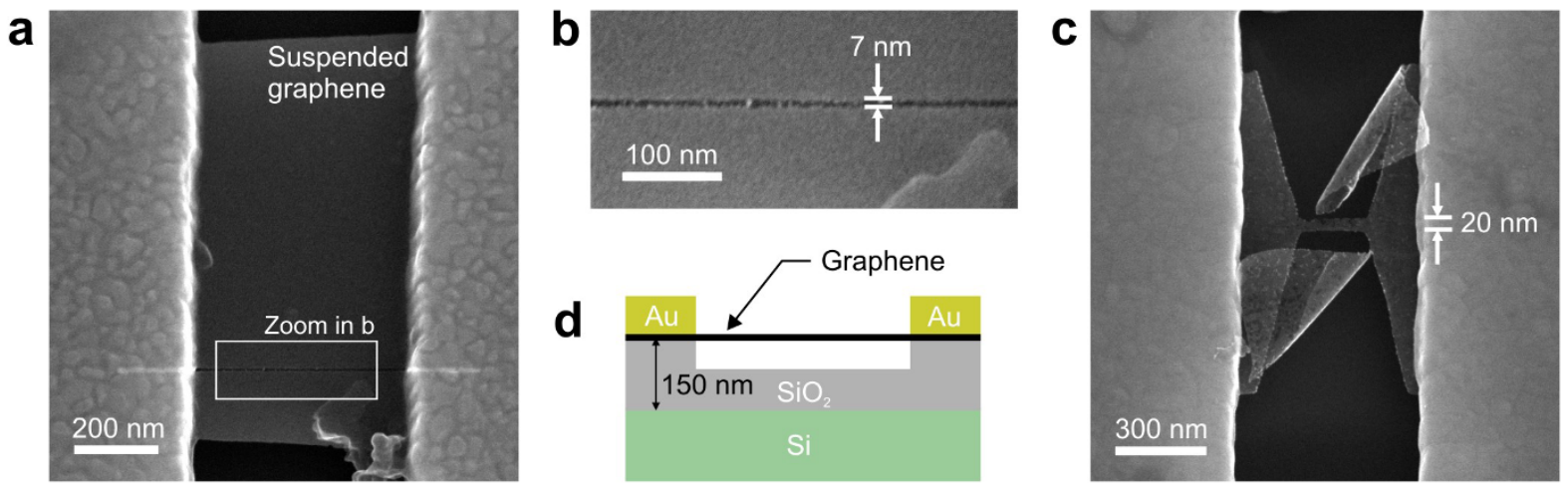

Figure 1 Nano-etching of suspended graphene: (a) An SEM picture of suspended graphene that has been cut by electron-beam-induced etching. Two gold contacts were placed at a distance of $500 \mathrm{~nm}$ and the graphene was suspended by removal of the supporting $\mathrm{SiO}_{2}$ by selective etching using a hydrofluoric acid solution (see methods). (b) Zoom-in of the line-cut of the suspended graphene layer, showing a minimum feature size of $\sim 7 \mathrm{~nm}$. (c) SEM picture of a suspended grapheme nano-ribbon with a width of $20 \mathrm{~nm}$. The rolled-up clippings are still visible, demonstrating the precision of the cuts. (d) Schematic picture of the sample structure with the suspended graphene. 

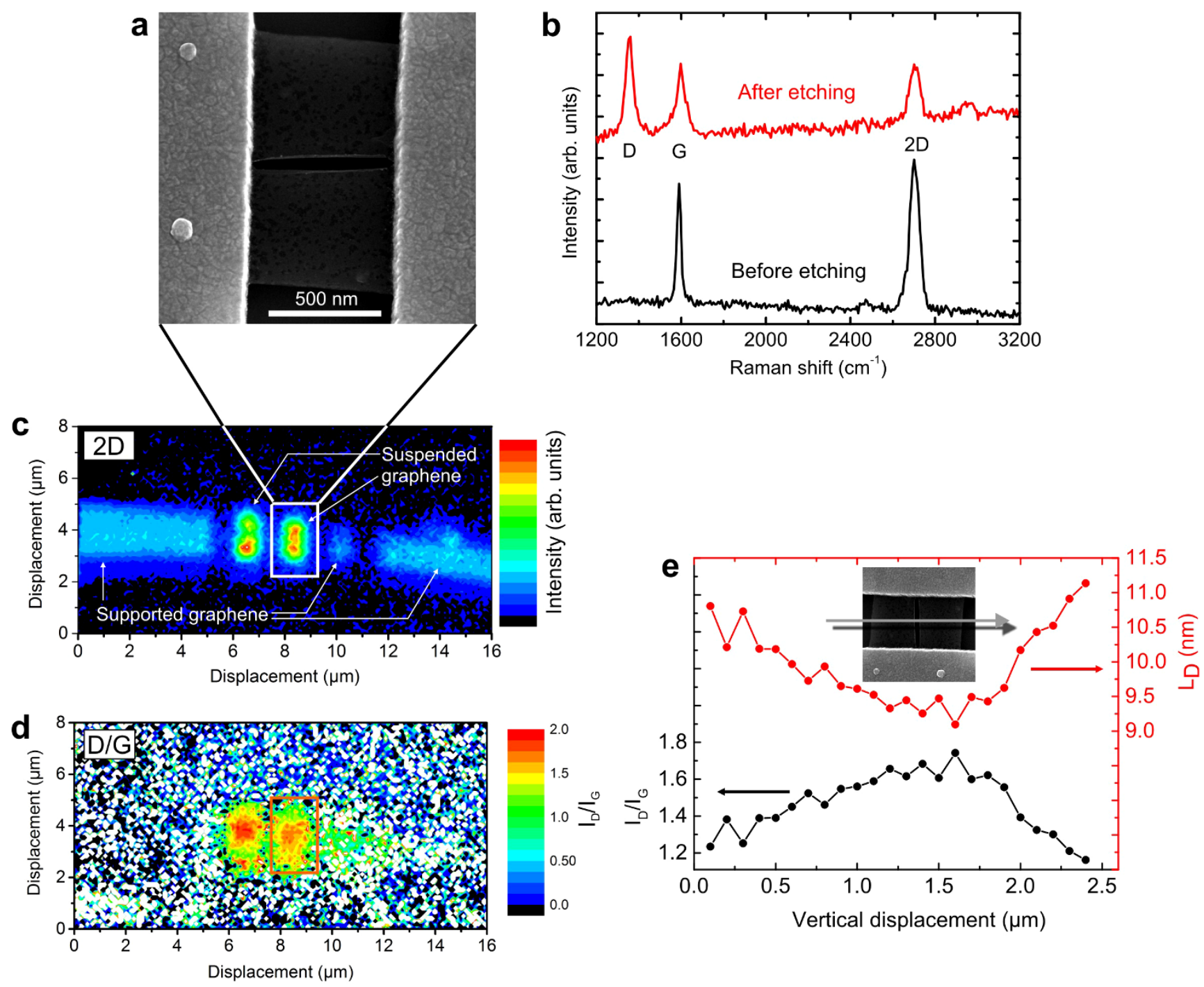

Figure 2 Quantification of point defect concentration by Raman spectroscopy: (a) SEM image of single line-cut through a suspended graphene layer. The etched graphene layer has been characterized concerning the point defect concentration by Raman spectroscopy. (b) A Raman spectrum before the gas-assisted etching (black line) and afterwards (red line). (c) A two-dimensional Raman map of the 2D peak across the entire structure where parts of the graphene is on the $\mathrm{SiO}_{2}$ surface (supported graphene) and parts are suspended between Au contacts. The marked area is the suspended part that has been etched and characterized with the SEM image in (a). The 2D intensity of the suspended graphene after etching is always higher than the 2D intensity of the supported layer on the left/right part of the Raman image. (d) The same 2D Raman map for the ratio between the peak intensity of the D and G peak: $\mathrm{I}_{\mathrm{D}} / \mathrm{I}_{\mathrm{G}}$. (e) Evaluation of the $\mathrm{I}_{\mathrm{D}} / \mathrm{I}_{\mathrm{G}}$ ratio (black line) from the $2 \mathrm{D}$ map in (d) across the etched area and the corresponding average distance of two point defects $\mathrm{L}_{\mathrm{D}}$ (red line).

cut. Hence, we are confident that we are still in the low defect density area. Using the evaluation method in Ref. 34, taking into account the laser wavelength of $532 \mathrm{~nm}$, and assuming only point defects, we obtain the average distance between point defects $\mathrm{L}_{\mathrm{D}}$ as a function of the displacement (red line in Fig. 2(e)). We find that $L_{D}$ has a maximum value of approximately $11 \mathrm{~nm}$ at the edges and decreases down to $9 \mathrm{~nm}$ in the middle of the graphene layer where the line-cut is located. The average value of $L_{D}=10 \mathrm{~nm}$ shows that while the etching does induce some damage, the resulting patterned graphene still maintains a high structural quality.

To also demonstrate the electronic quality of the etched graphene, transport measurements on a different suspended GNR with a width of $\sim 25 \mathrm{~nm}$ and a length of approx. $250 \mathrm{~nm}$ were conducted. By measuring the non-linear dc conductance as a function of gate bias and source-drain bias, we can estimate the energy of the transport gap in the graphene nano-ribbon ${ }^{13,14}$, displayed in Fig. 3(a). We have measured the conductance in a two-terminal measurement at $\mathrm{T}=$ $3 \mathrm{~K}$ with a source-measurement unit (SMU) connected to the source contact while the drain contact was grounded. The gate bias was applied by a second SMU between the highly doped Si substrate and the ground terminal. The measurement resolution is in the order of $0.1 \mu \mathrm{S}$ and therefore one order of magnitude lower resolution that in previously performed ac-measurements on graphene nano-ribbons on $\mathrm{SiO}_{2}{ }^{13}$. However, we can observe a gap region in Fig. 3(b) which extends between $\mathrm{Vg}=-25$ and $\mathrm{Vg}=30 \mathrm{~V}$ and is centred around zero gate bias, a situation that is not achieved easily for supported graphene for instance due to the unintentionally p-doping by a water film on the surface. We can roughly estimate the energy of the transport gap of the nano-ribbon from the diamond-shaped region, where the current is suppressed to our measurement limit of $0.1 \mu \mathrm{S}$, indicated in Fig. 3(a). As marked by the red arrow, the energy of the transport gap can be directly estimated from the value of the source-drain voltage $\mathrm{V}_{\text {sd }}$ at the vertex of the diamond. We obtain an energy of $\sim 60 \mathrm{meV}$, in good agreement within the error margin with the value obtained by Han et al. ${ }^{13,14}$ for a nano-ribbon of $25 \mathrm{~nm}$ width. 

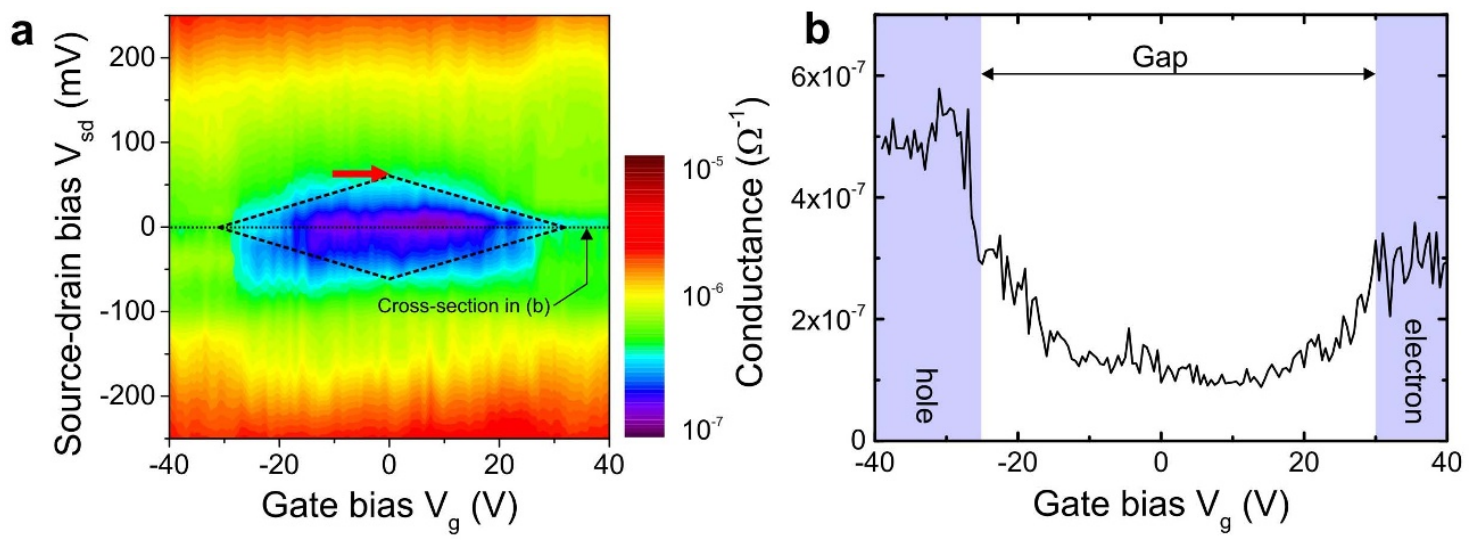

Figure 3 Transport measurement at $\mathrm{T}=3 \mathrm{~K}$ on a suspended graphene nanoribbon having a width of $\sim 25 \mathrm{~nm}$ : (a) The dc conductance $\mathrm{G}$ is shown as a function of source-drain $V_{s d}$ and gate bias $V_{g}$. The minimal conductance is limited by the experimental set-up and is in the order of $0.1 \mu \mathrm{S}$. The dark purple color indicates low conductance while the bright red color displays high conductance of the graphene nano-ribbon. From the conductance measurement of both gate bias $\mathrm{V}_{\mathrm{g}}$ and source-drain bias $\mathrm{V}_{\mathrm{sd}}$ we have drawn roughly a diamond shape that allows to estimate a value for the band-gap of approximately $\mathrm{E}_{\text {gap }}=60 \mathrm{meV}$ (indicated by the red arrow). (b) Conductance of the graphene nano-ribbon as a function of the gate bias $\mathrm{V}_{\mathrm{g}}$ at zero sourcedrain bias. An transport gap between $\mathrm{V}_{\mathrm{g}}=-25 \mathrm{~V}$ and $\mathrm{V}_{\mathrm{g}}=30 \mathrm{~V}$ is visible that is centered around zero gate bias.

\section{Conclusions}

In conclusion, we demonstrated the first gas-assisted nano-etching of suspended graphene with a minimum resolution of only $\sim 7 \mathrm{~nm}$ for line-cuts into the monolayer graphene. We showed with this method patterning of a $25 \mathrm{~nm}$ suspended graphene nanoribbon (GNR) and measured a transport gap in such a patterned GNR that is in good agreement with values for supported graphene found in the literature. We quantified the average distance of point defects by using 2D Raman maps and obtained a value that is in the order of $10 \mathrm{~nm}$, demonstrating the high quality of the etched graphene layer. We expect this technique to be of great value for nano-structuring of suspended graphene for future ballistic transport, nano-mechanical and nano-electronic devices.

\section{Methods}

Sample preparation. The graphene flakes used for the experiments presented in this work were fabricated by mechanical exfoliation on highly doped Si-substrates covered with a $300 \mathrm{~nm}$ thick layer of thermally grown $\mathrm{SiO}_{2}$. The graphene flakes are identified using optical microscopy and the layer thickness is determined by Raman spectroscopy and the optical contrast. The graphene flakes are contacted by $5 \mathrm{~nm}$ titanium and $100 \mathrm{~nm}$ gold after exfoliation by standard e-beam lithography. Afterwards, the Graphene was under-etched by submerging the samples in buffered oxide etch $\left(7: 1 \mathrm{NH}_{4} \mathrm{~F}\right.$ and $\left.\mathrm{HF}\right)$ for $100 \mathrm{~s}$, which removes $\sim 150 \mathrm{~nm}$ of $\mathrm{SiO}_{2}$ beneath the graphene. Finally the samples are dried in hot isopropyl alcohol, leaving a free standing grapheme sheet, which is clamped by the Ti/Au contacts, behind.

Gas assisted electron beam etching. We used a focused electron beam of a scanning electron microscope (SEM) in a combined dual-beam focused ion beam system (FEI NanoLab 600). The electron beam had a nominal resolution of $0.9 \mathrm{~nm}$ in a highresolution immersion mode. For the gas-assisted nano-etching process, the water vapor was directly injected by a gas-injection needle onto the sample surface while the electron beam was scanned over the graphene layer. After the water molecules from the vapor are adsorbed on the graphene, they are dissociated by the electron beam and the ionized oxygen atoms react with the carbon in the graphene layers to volatile carbon products, $\mathrm{CO}_{\mathrm{x}}{ }^{30}$, that are removed from the surface into the vacuum chamber.

Raman spectroscopy. We used a micro-photoluminescence ( $\mu$-PL) set-up in a confocal geometry with a long-distance objective having a magnification of $50 \mathrm{x}$ and a numerical aperture of $\mathrm{NA}=0.5$. This set-up has a spot size of approximately $1 \mu \mathrm{m}$. As an excitation source we used a frequency-doubled $\mathrm{Nd}: \mathrm{YVO}_{4}$ laser at a wavelength of $532 \mathrm{~nm}$. The Raman spectra were measured with a Czerny-Turner type spectrometer with a $1501 / \mathrm{mm}$ grating and a liquid-nitrogen cooled CCD in backscattering geometry. The resolution for all Raman spectra was $\sim 15$ wavenumbers. For the two-dimensional Raman maps at room temperature in Fig. 2, we placed the graphene sample on an $\mathrm{x}-\mathrm{y}$ stage with a step size in both directions of $100 \mathrm{~nm}$.

1. Novoselov, K. S. et al. Electric field effect in atomically thin carbon films. Science 306, 666-669 (2004)
2. Geim, A. K. \& Novoselov, K. S. The rise of graphene. Nature Mater. 6, 183-191 (2007).

3. Novoselov, K. S. et al. Two-dimensional gas of massless Dirac fermions in graphene. Nature 438, 197-200 (2005).

4. Bolotin, K., Ghahari, F., Shulman, M., Störmer, H. L. \& Kim, P. Observation of the fractional quantum Hall effect in graphene. Nature 462, 196-199 (2009).

5. Schwierz, F. Graphene transistors. Nature Nanotech. 5, 487-496 (2010).

6. Yan, Q. et al. Intrinsic current-voltage characteristics of graphene nanoribbon transistors and effect of edge doping. Nano Lett. 7, 1469-1473 (2007).

7. Areshkin, D. A. \& White, C. T. Building blocks for integrated graphene circuts. Nano Lett. 7, 3253-3259 (2007).

8. Du, X., Skachko, I., Barker, A. \& Andrei, E. Y. Approaching ballistic transport in suspended graphene. Nature Nanotech. 3, 491-495 (2008)

9. Ishigami, M., Chen, J. H., Cullen, W. G., Fuhrer, M. S. \& Williams, E. D. Atomic structure of graphene on $\mathrm{SiO}_{2}$. Nano Lett. 7, 1643-1648 (2007).

10. Dean, C. R. et al. Boron nitride substrates for high-quality graphene electronics. Nature Nanotech. 5, 722-726 (2010).

11. Bolotin, K. I., Sikes, K. J., Hone, J., Störmer, H. L. \& Kim, P. Temperaturedependent transport in suspended graphene. Phys. Rev. Lett. 101, 096802 (2008).

12. Molitor, F. et al. Local gating of a graphene Hall bar by graphene side gates. Phys. Rev. B 76, 245426 (2007).

13. Han, M. Y., Özyilmaz, B., Zhang, Y. \& Kim, P. Energy band-gap engineering of graphene nanoribbons. Phys. Rev. Lett. 98, 206805 (2007).

14. Han, M. Y., Brant, J. C. \& Kim, P. Electron Transport in disordered graphene nanoribbons. Phys. Rev. Lett. 104, 056801 (2010).

15. Güttinger, J. et al. Transport through graphene quantum dots. Rep. Prog. Phys. 75, 126502 (2012).

16. Ponomarenko, L. A. et al. Chaotic Dirac billiard in graphene quantum dots. Science 320, 356-358 (2008).

17. Archanjo, B. S. et al. The use of a $\mathrm{Ga}^{+}$focused ion beam to modify graphene for device applications. Nanotechnology 23, 255305 (2012).

18. Bell, D. C. et al. Precision cutting and patterning of graphene with helium ions. Nanotechnology 20, 455301 (2009).

19. Lemme, M. C., et al. Etching of Graphene devices with a helium ion beam. ACS Nano 9, 2674-2676 (2009).

20. Krasheninnikov, A. V. \& Nordlund, K. Ion and electron irradiation-induced effects in nanostructred materials. J. Appl. Phys. 107, 071301 (2010).

21. Fischbein, M. D. \& Drndić, M. Electron beam nanosculpting of suspended graphene sheets. Appl. Phys. Lett. 93, 113107 (2008).

22. Xu, Q. et al. Controllable Atomic Scale Patterning of Freestanding Monolayer Graphene at Elevated Temperature. ACS Nano 7, 1566-1572 (2013).

23. Tapasztó, L., Dobrik, G., Lambin, P. \& Biró, L. P. Tailoring the atomic structure of graphene nanoribbons by scanning tunnelling microscope lithography. Nature Nanotech. 3, 397-401 (2008).

24. Masubuchi, S., Ono, M., Yoshida, K., Hirakawa, K. \& Machida, T. Fabrication of graphene nanoribbon by local anodic oxidation lithography using atomic force microscope. Appl. Phys. Lett. 94, 082107 (2009).

25. Byun, I.-S. et al. Nanoscale lithography on monolayer graphene using hydrogenation and oxidation. ACS Nano 5, 6417-6424 (2011).

26. Randolph, S. J., Fowlkes, J. D. \& Rack, P. D. Focused, nanoscale electron-beaminduced deposition and etching. Crit. Rev. Solid State 31, 55-89 (2006).

27. Utke, I., Hoffmann, P. \& Melngailis, J. Gas-assisted focused electron beam and ion beam processing and fabrication. J. Vac. Sci. Technol. B 26, 1197-1276 (2008). 
28. Matsui, S. \& Katsumi, H. Direct writing onto Si by electron beam stimulated etching. Appl. Phys. Lett. 51, 1498-1499 (1987).

29. Ganczarczyk, A., Geller, M. \& Lorke, A. $\mathrm{XeF}_{2}$ gas-assisted focused-electron-beam induced etching of GaAs with 30 nm-resolution. Nanotechnology 22, 045301 (2011).

30. Spinney, P. S., Howitt, D. G., Collins, S. D. \& Smith, R. L. Electron beam stimulated oxidation of carbon. Nanotechnology 20, 456301 (2009).

31. Thiele, C. et al. Electron-beam-induced direct etching of graphene. Carbon 64, 84-91 (2013).

32. Ni, Z. H. et al. Probing charged impurities in suspended graphene using raman spectroscopy. ACS Nano 3, 569 (2009).

33. Ferrari, A. C. \& Basko, D. M. Raman spectroscopy as a versatile tool for studying the properties of graphene. Nature Nanotech. 8, 235-246 (2013).

34. Cancado, L. G. et al. Quantifying Defects in Graphene via Raman Spectroscopy at Different Excitation Energies. Nano Lett. 11, 3190-3196 (2011).

\section{Author contributions}

D.B. and G.P. carried out the Raman spectroscopy and analyzed the results. B.S., J.S. and A.G. fabricated the graphene samples and made the transport measurements. A.L. and M.G. supervised the work, analysed the data and wrote the manuscript.

\section{Additional information}

Supplementary information accompanies this paper at http://www.nature.com/ scientificreports

Competing financial interests: The authors declare no competing financial interests.

How to cite this article: Sommer, B. et al. Electron-beam induced nano-etching of suspended graphene. Sci. Rep. 5, 7781; DOI:10.1038/srep07781 (2015).

(c) (1) (9) This work is licensed under a Creative Commons Attribution-NonCommercialcc. ${ }_{\mathrm{BY}} \mathrm{NC}$ ND NoDerivs 4.0 International License. The images or other third party material in this article are included in the article's Creative Commons license, unless indicated otherwise in the credit line; if the material is not included under the Creative Commons license, users will need to obtain permission from the license holder in order to reproduce the material. To view a copy of this license, visit http:// creativecommons.org/licenses/by-nc-nd/4.0/ 